\title{
Effect of palmitoylethanolamide on inflammatory and neuropathic pain in rats
}

\author{
Tai-Kyung Seol, Wonho Lee, Sunah Park, Kyu Nam Kim, Tae Yeon Kim, \\ You $\mathrm{Na} \mathrm{Oh}$, and Jong Hun Jun \\ Department of Anesthesiology and Pain Medicine, Hanyang University College of Medicine, Seoul, Korea
}

Background: A growing body of evidence suggests that neuroinflammation, which is characterized by infiltration of immune cells, activation of mast cells and glial cells, and production of inflammatory mediators in the peripheral and central nervous systems, plays an important role in the induction and maintenance of chronic pain. Palmitoylethanolamide (PEA), which is a type of $\mathrm{N}$-acylethanolamide and a lipid, has an anti-inflammatory effect. Relative to the anti-inflammatory effect, little is known about its analgesic effect in chronic pain. This study aimed to determine whether PEA relieves chronic inflammatory and neuropathic pain.

Methods: Male Sprague-Dawley rats were injured by transection of the left L5 and L6 spinal nerves to induce neuropathic pain or were injected with monoiodoacetic acid into the synovial cavity of knee joints to induce inflammatory pain. To assess the degree of pain, two kinds of stimuli - pressing von Frey filaments and wetting with acetone - were applied to the plantar surface of the rat to measure mechanical and cold sensitivity, respectively. Pain was measured by assessing behavioral responses, including paw withdrawal response threshold and paw withdrawal frequency upon stimulation.

Results: Neuropathic pain caused by spinal nerve transection (SNT) decreased the mechanical threshold and increased the frequency of response to acetone application. But, cold allodynia caused by SNT did not decrease the withdrawal frequency. Mechanical hyperalgesia caused by chronic inflammation was significantly reduced by both intraperitoneal and intra-articular injections of PEA.

Conclusions: These outcomes revealed that PEA might be effective in relieving inflammatory and neuropathic pain, especially pain induced by mechanical hyperalgesia, but not cold allodynia.

Key Words: Inflammatory pain, Neuropathic pain, Palmitoylethanolamide.

Corresponding author: Jong Hun Jun, M.D.

Department of Anesthesiology and Pain Medicine, Hanyang University College of Medicine, 222, Wangsimni-ro, Seongdong-gu, Seoul 04763 , Korea

Tel: 82-2-2290-8680, Fax: 82-2-2299-8692

Email: jhjun@hanyang.ac.kr

This is a thesis for a Doctor's degree by Tai-Kyung Seol.

Received: May 24, 2016.

Revised: October 6, 2016 (1st); January 5, 2017 (2nd); June 4, 2017 (3rd). Accepted: June 5, 2017.

Korean J Anesthesiol 2017 October 70(5): 561-566

https://doi.org/10.4097/kjae.2017.70.5.561

\section{Introduction}

Palmitoylethanolamide (PEA), an endogenous fatty acid amide with the chemical structure of $\mathrm{N}$-(2-hydroxyethyl) esadecanamides, was regarded as an autacoid. Specifically, it is known mostly as an anti-inflammatory agent due to the downregulation of mediator release from mast cells, monocytes and macrophages [1-4]. It is assumed that PEA has potent immunoregulatory properties. Recently, it was proven that PEA possesses powerful abilities in the regulation of complex systems involved in the inflammatory response, pruritus, and neurogenic and neuropathic pain [5].

(c) This is an open-access article distributed under the terms of the Creative Commons Attribution Non-Commercial License (http://creativecommons.org/ licenses/by-nc/4.0/), which permits unrestricted non-commercial use, distribution, and reproduction in any medium, provided the original work is properly cited. 
Commonly, PEA is believed to participate in endogenous defensive mechanisms initiated by various forms of tissue damage, or stimulation of inflammatory responses and nociceptive fibers in the body. PEA, which consists of $\mathrm{N}$-acylethanolamides and lipids, has been known to have anti-inflammatory effects. Unlike its anti-inflammatory effect, little known about its analgesic effect in chronic pain.

There has been a lot of research suggesting that PEA has relieved the degree of pain without any harmful side effects in humans [6-9]. PEA might have anti-inflammatory and analgesic effects under certain circumstances associated with inflammation and pain in animal experiments.

PEA has an extensive spectrum of analgesia and these effects has already been tested in many different types of pain models. It alleviates pain behaviors evoked by formalin [10], magnesium sulfate [11], carrageenan [2,12], nerve growth factor [13], and turpentine $[14,15]$. Additionally, PEA was reported to inhibit hyperalgesia after sciatic nerve ligation in a model of neuropathic pain [16]. PEA could be involved in the endogenous regulation of nociception, as PEA-produced analgesia is fast and occurs before its anti-inflammatory actions [10]. However, the specific analgesic mechanism of PEA is currently unknown.

When monoiodoacetic acid (MIA) is injected locally into joints, it promptly produces inflammation, interrupts chondrocyte metabolism, and then induces cartilage degeneration. Furthermore, the osteoarthritis induced by MIA in rats is similar to human osteoarthritis with respect to the cartilage and bone pathological features and can be used as a minimally invasive animal model.

The present study was executed to clarify whether PEA could alleviate neuropathic pain from spinal nerve transection (SNT) and inflammatory pain from intra-articular MIA administration in a different ways than that used earlier.

\section{Materials and Methods}

The Institutional Animal Investigation Committee approved every step of the present study. We used 154 male SpragueDawley rats, weighing 130-160 g, in this experiment and divided them into groups of 5 rats per cage. Each cage was filled with unlimited food and water under a $12 \mathrm{~h}$ light/dark cycle. The neuropathic pain model used in this experiment was conducted as SNT derived from the original spinal nerve injury model of spinal nerve ligation $[17,18]$. Under anesthesia with $2-3 \%$ enflurane, we incised the back skin, removed the left transverse process of the L6 vertebra, and subsequently transected the left L5 and L6 spinal nerves. To create an inflammatory injury, $50 \mu \mathrm{l}$ of $4 \%$ MIA was administered into the synovial cavity of left knee joint.

Two types of stimuli, contact with von Frey filaments and wetting with acetone, were used on the plantar surface of rats to estimate pain by measuring mechanical and cold sensitivity, respectively. The withdrawal response to each stimulus was quantified. To facilitate the process, rats were put on a metal mesh floor under custom-made transparent acrylic cells and acclimated for $10 \mathrm{~min}$ or more.

To identify mechanical sensitivity, we pressed the von Frey filament onto the middle of the plantar surface of the left hindpaw using an up and down motion with bending forces of 0.65 , $1.05,1.56,2.60,4.89,6.16,8.40,15.25$, and $21.75 \mathrm{~g}$ beginning at a filament of $6.16 \mathrm{~g}$ (the von Frey filament number is 4.74 ), and the $50 \%$ paw withdrawal response threshold (PWT) was calculated as described by Chaplan et al. [19]. The filament of von Frey number 4.74 (its bending force is $6.16 \mathrm{~g}$ ) was perpendicularly applied to the plantar surface. If a paw withdrawal response was seen, a filament with the next lower force was applied. If a paw withdrawal response was not seen, a filament with the next higher force was applied. We repeated these procedures until a series of 6 stimulation-response records were recorded. A bending force of $21.75 \mathrm{~g}$ was chosen as the upper limit for testing, because the filaments with a bending force of $10 \%$ or more of body weight tended to passively raise the entire limb rather than to induce an active brisk withdrawal.

For evaluation of cold sensitivity, vigorous withdrawals were counted as a response. For this, 1-3 drops of acetone were applied with a $16 \mathrm{G}$ blunt-ended stainless needle, and acetone was applied 5 times on the plantar of the left hindpaw at intervals of 2 or more min at room temperature. The needle did not touch the plantar surface of the hindpaw. Data are represented as paw withdrawal frequency (PWF) [(number of response / number of trials) $\times 100$ ].

To determine the effect of PEA on neuropathic and inflammatory pain, PEA was injected intraperitoneally (vehicle, 2, 5, 10 $\mathrm{mg}$ ) or intra-articularly (vehicle, 0.05, 0.1, $0.2 \mathrm{mg}$ ) 2 weeks after SNT or MIA injection in each experiment model. The challenge dose of PEA was determined in a previous experiment and in our pilot study. PEA was dissolved in $10 \%$ ethanol and $90 \%$ normal saline. The effect of PEA in each experimental model was evaluated $10 \mathrm{~min}, 20 \mathrm{~min}, 40 \mathrm{~min}, 1 \mathrm{~h}, 2 \mathrm{~h}, 4 \mathrm{~h}, 6 \mathrm{~h}$, and $24 \mathrm{~h}$ after injection as PWT and PWF.

Rats were sacrificed using high-dose thiopental sodium anesthesia immediately after the experiment.

Statistical analyses were performed using Jandel Scientific SigmaStat for Windows, version 3.5 with one-way ANOVA, followed by the Dunnett's test for post hoc comparisons between points or Student's $t$ test. Statistical significance was determined at a $\mathrm{P}$ value less than 0.05 . Data are expressed as mean \pm SEM. 


\section{Results}

SNT rats showed a marked decrease in PWT. PWT reached a plateau (4 g) 2-4 days after the injury and was maintained at this level for 3 weeks or longer (Fig. 1). The PWT increased significantly from $2.0 \pm 0.2 \mathrm{~g}$ to $3.0 \pm 0.2 \mathrm{~g}$ for PEA $2 \mathrm{mg}$ and from 1.8 $\pm 0.2 \mathrm{~g}$ to $3.0 \pm 0.6 \mathrm{~g}$ for PEA $5 \mathrm{mg}, 10 \mathrm{~min}$ after intraperitoneal PEA injection when compared to the vehicle group. Additionally, PWT increased significantly to $3.5 \pm 1.0 \mathrm{~g}, 3.3 \pm 0.5 \mathrm{~g}, 3.5$ $\pm 0.5 \mathrm{~g}$, and $2.5 \pm 0.5 \mathrm{~g}$ at $10 \mathrm{~min}, 20 \mathrm{~min}, 40 \mathrm{~min}$, and $1 \mathrm{~h}$ after intraperitoneal injection of $10 \mathrm{mg}$ of PEA (Fig. 2). Mechanical hyperalgesia from SNT was significantly alleviated by intraperi-

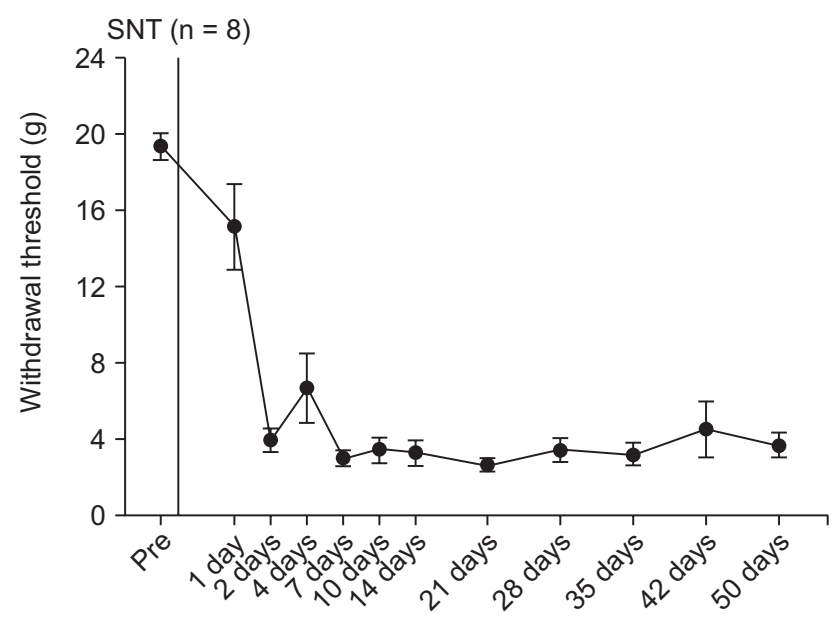

Fig. 1. Changes in paw withdrawal threshold in response to mechanical stimuli with von Frey filaments after spinal nerve transection (SNT) in 8 rats.

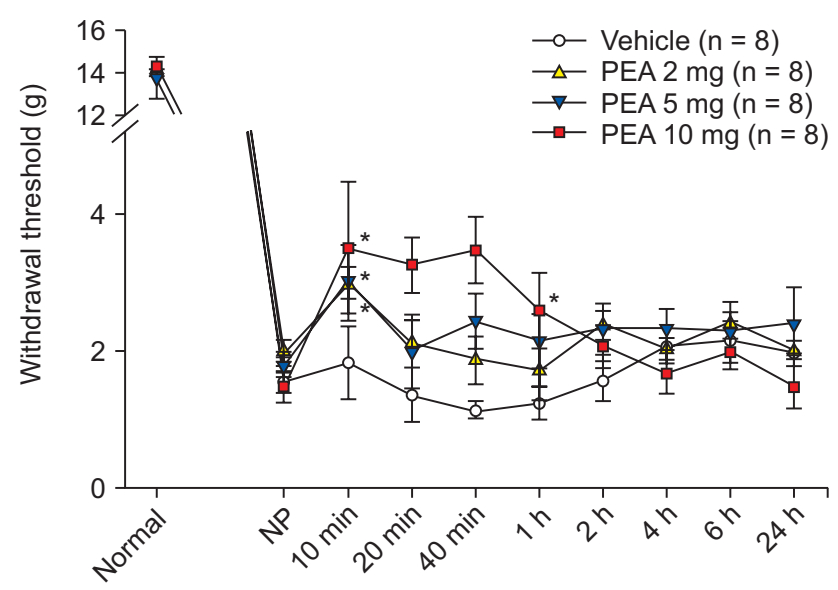

Fig. 2. Effects of palmitoylethanolamide (PEA) on mechanical hyperalgesia induced by spinal nerve transection after establishment of neuropathic pain (NP, 14 days after SNT). PEA was injected intraperitoneally. NP signifies a hyperalgesia state just before PEA injection. Numeric time signifies time after PEA injection. *Indicate values significantly different from the vehicle group. toneal PEA. SNT rats showed an increase in PWF after 1-4 days, and this increase was maintained for 3 weeks (Fig. 3). However, we did not observe a significant alleviation of cold allodynia from SNT by any dose of PEA tested (Fig. 4).

We observed inflammatory pain caused by MIA, which decreased the mechanical threshold. MIA intra-articular injection resulted in a marked decrease in PWT. PWT reached a plateau 1 day after injection and was maintained at a level not exceeding 5 $\mathrm{g}$ for 3 weeks (Fig. 5).

PWT increased significantly to $2.4 \pm 0.4 \mathrm{~g}$ and $2.6 \pm 0.3 \mathrm{~g}$ at $1 \mathrm{~h}$ and $2 \mathrm{~h}$ after intraperitoneal injection of $5 \mathrm{mg}$ of PEA; prior to injection, the PWT was $1.3 \pm 0.1 \mathrm{~g}$. PWT also increased sig-

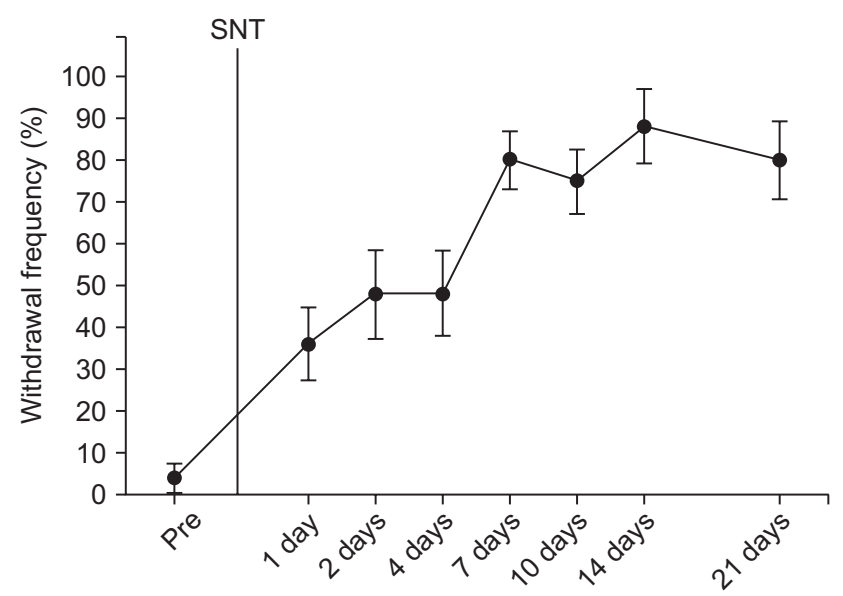

Fig. 3. Changes in withdrawal frequency induced by cold stimuli with acetone application after spinal nerve transection (SNT) in 8 rats.

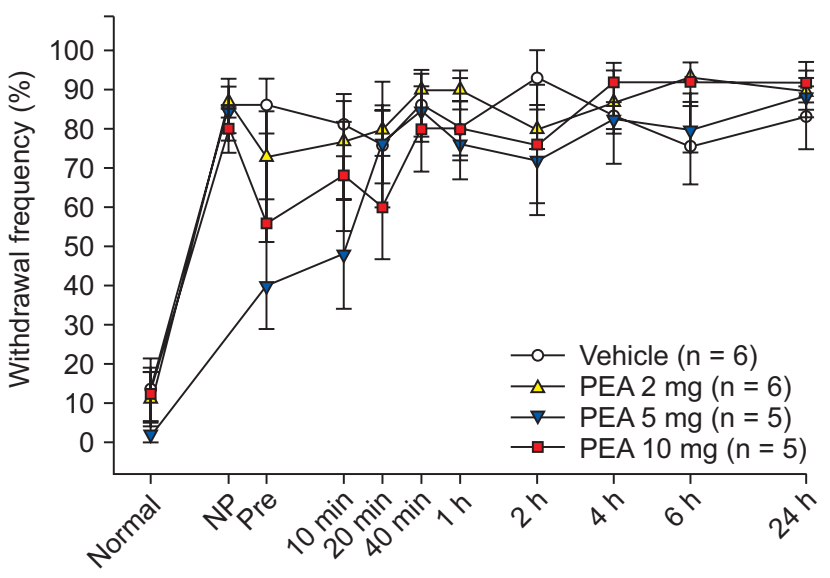

Fig. 4. Effects of palmitoylethanolamide (PEA) on cold allodynia induced by spinal nerve transection (SNT) after establishment of neuropathic pain (NP, 14 days after SNT). PEA was injected intraperitoneally. NP signifies a hyperalgesia state just before PEA injection. Numeric time signifies time after PEA injection. 


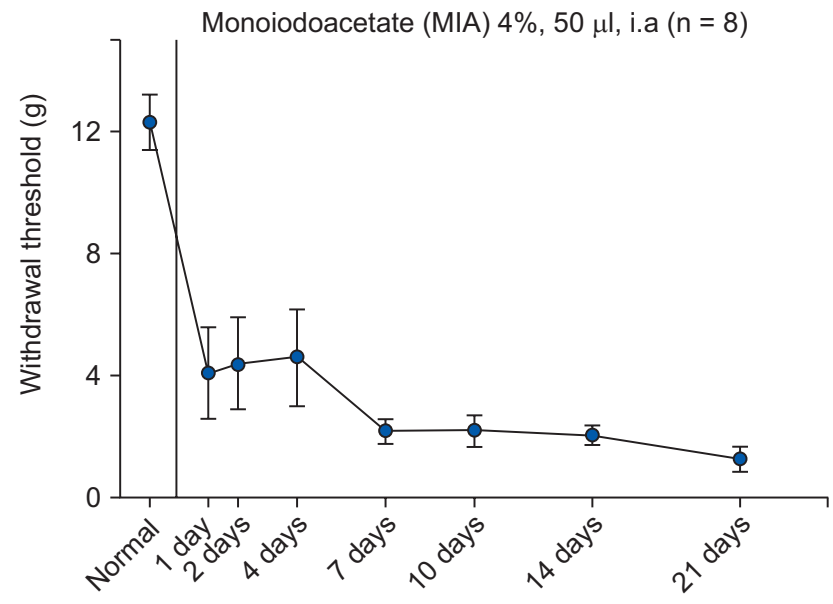

Fig. 5. Changes in withdrawal threshold for the paw withdrawal mechanical response induced by intra-articular injection of $50 \mu \mathrm{l}$ (i.a.) of $4 \%$ monoiodoacetate (MIA) in 8 rats.

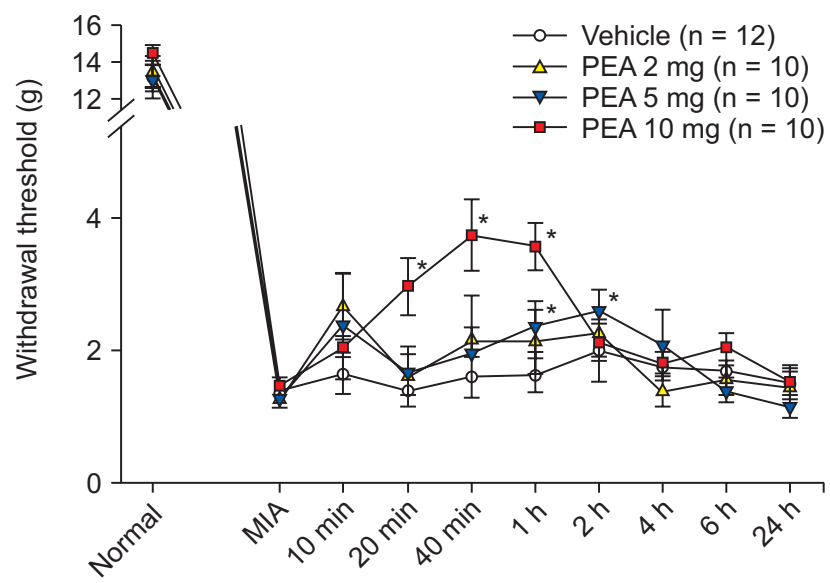

Fig. 6. Effect of intraperitoneal palmitoylethanolamide (PEA) on inflammatory hyperalgesia induced by intra-articular monoiodoacetate (MIA) injection. MIA signifies a hyperalgesia state just before PEA injection. Numeric time signifies time after PEA injection. ${ }^{*}$ Indicate values significantly different from the vehicle group.

nificantly to $3.0 \pm 0.4 \mathrm{~g}, 3.7 \pm 0.5 \mathrm{~g}$, and $3.6 \pm 0.4 \mathrm{~g}$ at $20 \mathrm{~min}$, $40 \mathrm{~min}$, and $1 \mathrm{~h}$ after intraperitoneal injection of $10 \mathrm{mg}$ of PEA; the pre-injection PWT was $1.5 \pm 0.1 \mathrm{~g}$ (Fig. 6). PWT increased significantly to $2.2 \pm 0.5 \mathrm{~g}$ and $2.2 \pm 0.4 \mathrm{~g}$ at 10 and $40 \mathrm{~min}$ after intra-articular injection of $0.1 \mathrm{mg}$ of PEA, compared to vehicle. PWT also increased significantly to $2.2 \pm 0.6 \mathrm{~g}, 1.4 \pm 0.2 \mathrm{~g}$, and 1.4 $\pm 0.2 \mathrm{~g}$ at $10 \mathrm{~min}, 40 \mathrm{~min}$, and $1 \mathrm{~h}$ after intra-articular injection of $0.2 \mathrm{mg}$ of PEA, compared to vehicle (Fig. 7). Both intraperitoneal and intra-articular injection of PEA significantly relieved mechanical hyperalgesia from chronic inflammation.

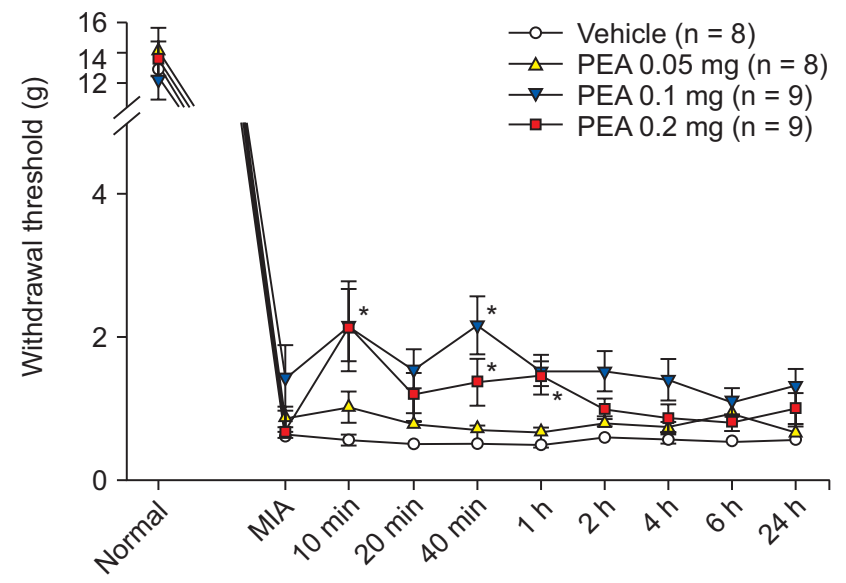

Fig. 7. Effect of intra-articular palmitoylethanolamide (PEA) on inflammatory hyperalgesia induced by intra-articular monoiodoacetate (MIA) injection. MIA signifies a hyperalgesia state just before PEA injection. Numeric time signifies time after PEA injection. *Indicate values significantly different from the vehicle group.

\section{Discussion}

The purpose of the present study was to determine if PEA could reduce neuropathic and inflammatory pain. SNT resulted in a constant reduction of PWT, one of the signs of neuropathic and inflammatory pain. Generally, the spinal nerve ligation model is used to induce chronic neuropathic pain. In our study, the L5 and L6 spinal nerves were transected rather than ligated to produce neuropathic pain. Compared to other studies on nerves, we observed behavioral signs of pain very similar to those seen in previous studies of SNT rats [17,18]. Therefore, SNT might be the method of choice for causing neuropathic pain given the advantages of a significantly easier operation and less mechanical irritation than ligation.

PEA has brought about outcomes including analgesia in acute and inflammatory pain $[10,12,15]$, suppression of food intake [20], decreased gastrointestinal motility [10], suppression of cancer cell proliferation [21], and neuroprotection [22]. Additionally, it was demonstrated that pain hypersensitivity resulting from sciatic nerve constriction (chronic constriction injury, CCI) in rats is related to a considerable decrement of endogenous PEA level in the spinal cord and in brain areas directly or indirectly concerned with nociception [23]. Consequently, it is possible that this lipid could be associated with pain-related behaviors. It has been previously demonstrated that a single injection of PEA [16] or its analogue palmitoylallylamide [24], which supplies the lacking endogenous PEA, significantly improved neuropathic pain in a rat partial sciatic nerve injury model.

In experimental data reported by Calignano et al. [10], it was shown that arachidonylethanolamide (AEA) alleviates pain behavior induced by chemical injury to cutaneous tis- 
sue by interaction with cannabinoid 1 receptors located in the periphery. They also reported a similar effect of PEA, which is released together with AEA from a common phospholipid precursor, which operates via peripheral cannabinoid 2 (CB2)-like receptors. As demonstarted in different acute pain models like formalin [15], acetic acid, kaolin, $\mathrm{MgSO}_{4}$, and capsaicin and in hot plate [11] and tail flick tests [25], AEA has a powerful antinociceptive action. It has also been shown to be an effective antihyperalgesic compound under inflammatory pain conditions and to suppress turpentine-evoked viscerovisceral hyper-reflexia of the urinary bladder [15], carrageenin-induced thermal hyperalgesia [25], and mechano-nociceptive hyperalgesia in Freund's complete adjuvant-induced arthritis [26]. The other endogenous fatty acid ethanolamide, PEA, a selective agonist on peripheral CB2-like receptors, has been shown to inhibit carrageenininduced mechanical hyperalgesia [2] and formalin- [15], acetic acid-, kaolin- and $\mathrm{MgSO}_{4}$-induced acute pain; however, it has no effect on capsaicin-evoked and thermal nociception [11]. These results verify that endogenous palmitoylethanolamide is involved in the intrinsic control of pain induction. They also proposed that the putative receptor site activated by palmitoylethanolamide might represent a new target for peripherally acting analgesic drugs.

In this study, we were unable to demonstrate the analgesic effect of PEA on inflammatory pain through cold allodynia because cold allodynia did not reduce inflammatory pain upon intra-articular injection of MIA (data not shown).

As supported by the evidence, one distinct neuropathic pain symptom, i.e., cold allodynia, is generated by a differential loss of one type of afferent, the A $\delta$-cold specific fibers [27]. Blocking $A \delta$-fibers with a tourniquet in healthy subjects brings about an impairment of innocuous cool sensation (cold hypoesthesia), which is mediated by these fibers. Paradoxically, a decrease in the threshold for cold pain (cold hyperalgesia) was observed, which was mediated by polymodal C-nociceptors (CMH-fibers).
Hot and burning pain could be sensed. Obviously, the A $\delta$-input provides cold sensation as well as inhibiting the central responses to the $\mathrm{C}$-nociceptors activated by cold stimuli. The threshold for a $\mathrm{C}$-fiber-evoked sensation is reduced as $\mathrm{A} \delta$-conduction is blocked, and this inhibition vanishes. The quantity of the sensation (burning pain) is relative to the C-fiber input. Ochoa and Yarnitsky [27] reported on neuropathic pain patients with a disproportionate loss of $\mathrm{A} \delta$-axons and relative sparing of $\mathrm{C}$-fibers.

The reason we did not see cold allodynia upon intra-articular injection of MIA in our study might be that $\mathrm{A} \delta$-conduction could not be blocked appropriately, and that central inhibition was not lost. By contrast, unlike cold allodynia, mechanical hyperalgesia was alleviated by PEA in our neuropathic model. This suggests that SNT might not result in disproportionate loss of $\mathrm{A} \delta$-axons and relative sparing of $\mathrm{C}$-fibers unlike other neuropathic models, because SNT might result in more complete discontinuity of the nerve root compared to ligation. In addition, these differences might be due to the wide variety of mechanisms of neuropathic and inflammatory pain; consequently, the response to a drug might also vary widely depending on the mechanism of action. Based on these differences, more research will be needed to gain a full understanding.

Mechanical hyperalgesia from inflammation by MIA was alleviated by either intraperitoneal or intra-articular PEA. The dose used for intra-articular injection of PEA was less than that for intraperitoneal injection. This result suggests that the putative site activated by PEA might be peripheral as well as central. Intra-articular PEA might have fewer side effects than intraperitoneal PEA as the required dosage is less.

These outcomes revealed that PEA might be effective in relieving inflammatory and neuropathic pain, especially due to mechanical hyperalgesia, but not cold allodynia. The lack of an effect on neuropathic pain and inflammatory cold and heat pain should be further investigated with respect to pain mechanisms.

\section{References}

1. Facci L, Dal Toso R, Romanello S, Buriani A, Skaper SD, Leon A. Mast cells express a peripheral cannabinoid receptor with differential sensitivity to anandamide and palmitoylethanolamide. Proc Natl Acad Sci U S A 1995; 92: 3376-80.

2. Mazzari S, Canella R, Petrelli L, Marcolongo G, Leon A. N-(2-hydroxyethyl)hexadecanamide is orally active in reducing edema formation and inflammatory hyperalgesia by down-modulating mast cell activation. Eur J Pharmacol 1996; 300: 227-36.

3. Ross RA, Brockie HC, Pertwee RG. Inhibition of nitric oxide production in RAW 264.7 macrophages by cannabinoids and palmitoylethanolamide. Eur J Pharmacol 2000; 401: 121-30.

4. Berdyshev EV. Cannabinoid receptors and the regulation of immune response. Chem Phys Lipids 2000; 108: 169-90.

5. Di Marzo V, Melck D, De Petrocellis L, Bisogno T. Cannabimimetic fatty acid derivatives in cancer and inflammation. Prostaglandins Other Lipid Mediat 2000; 61: 43-61.

6. Kopsky DJ, Hesselink JM. Multimodal stepped care approach with acupuncture and PPAR-a agonist palmitoylethanolamide in the treatment of a patient with multiple sclerosis and central neuropathic pain. Acupunct Med 2012; 30: 53-5.

7. Cobellis L, Castaldi MA, Giordano V, Trabucco E, De Franciscis P, Torella M, et al. Effectiveness of the association micronized 
N-Palmitoylethanolamine (PEA)-transpolydatin in the treatment of chronic pelvic pain related to endometriosis after laparoscopic assessment: a pilot study. Eur J Obstet Gynecol Reprod Biol 2011; 158: 82-6.

8. Calabrò RS, Gervasi G, Marino S, Mondo PN, Bramanti P. Misdiagnosed chronic pelvic pain: pudendal neuralgia responding to a novel use of palmitoylethanolamide. Pain Med 2010; 11: 781-4.

9. Bacci C, Cassetta G, Emanuele B, Berengo M. Randomized split-mouth study on postoperative effects of palmitoylethanolamide for impacted lower third molar surgery. ISRN Surg 2011; 2011: 917350.

10. Calignano A, La Rana G, Giuffrida A, Piomelli D. Control of pain initiation by endogenous cannabinoids. Nature 1998; $394: 277-81$.

11. Calignano A, La Rana G, Piomelli D. Antinociceptive activity of the endogenous fatty acid amide, palmitylethanolamide. Eur J Pharmacol 2001; 419: 191-8.

12. Conti S, Costa B, Colleoni M, Parolaro D, Giagnoni G. Antiinflammatory action of endocannabinoid palmitoylethanolamide and the synthetic cannabinoid nabilone in a model of acute inflammation in the rat. Br J Pharmacol 2002; 135: 181-7.

13. Farquhar-Smith WP, Rice AS. A novel neuroimmune mechanism in cannabinoid-mediated attenuation of nerve growth factor-induced hyperalgesia. Anesthesiology 2003; 99: 1391-401.

14. Farquhar-Smith WP, Rice AS. Administration of endocannabinoids prevents a referred hyperalgesia associated with inflammation of the urinary bladder. Anesthesiology 2001; 94: 507-13.

15. Jaggar SI, Hasnie FS, Sellaturay S, Rice AS. The anti-hyperalgesic actions of the cannabinoid anandamide and the putative CB2 receptor agonist palmitoylethanolamide in visceral and somatic inflammatory pain. Pain 1998; 76: 189-99.

16. Helyes Z, Németh J, Thán M, Bölcskei K, Pintér E, Szolcsányi J. Inhibitory effect of anandamide on resiniferatoxin-induced sensory neuropeptide release in vivo and neuropathic hyperalgesia in the rat. Life Sci 2003; 73: 2345-53.

17. Lee SE, Kim JH. Involvement of substance P and calcitonin gene-related peptide in development and maintenance of neuropathic pain from spinal nerve injury model of rat. Neurosci Res 2007; 58: 245-9.

18. Kim SH, Chung JM. An experimental model for peripheral neuropathy produced by segmental spinal nerve ligation in the rat. Pain 1992; 50: 355-63.

19. Chaplan SR, Bach FW, Pogrel JW, Chung JM, Yaksh TL. Quantitative assessment of tactile allodynia in the rat paw. J Neurosci Methods 1994; 53: 55-63.

20. Rodríguez de Fonseca F, Navarro M, Gómez R, Escuredo L, Nava F, Fu J, et al. An anorexic lipid mediator regulated by feeding. Nature 2001; 414: 209-12.

21. De Petrocellis L, Bisogno T, Ligresti A, Bifulco M, Melck D, Di Marzo V. Effect on cancer cell proliferation of palmitoylethanolamide, a fatty acid amide interacting with both the cannabinoid and vanilloid signalling systems. Fundam Clin Pharmacol 2002; 16: 297-302.

22. Sheerin AH, Zhang X, Saucier DM, Corcoran ME. Selective antiepileptic effects of N-palmitoylethanolamide, a putative endocannabinoid. Epilepsia 2004; 45: 1184-8.

23. Petrosino S, Palazzo E, de Novellis V, Bisogno T, Rossi F, Maione S, et al. Changes in spinal and supraspinal endocannabinoid levels in neuropathic rats. Neuropharmacology 2007; 52: 415-22.

24. Wallace VC, Segerdahl AR, Lambert DM, Vandevoorde S, Blackbeard J, Pheby T, et al. The effect of the palmitoylethanolamide analogue, palmitoylallylamide (L-29) on pain behaviour in rodent models of neuropathy. Br J Pharmacol 2007; 151: 1117-28.

25. Richardson JD, Aanonsen L, Hargreaves KM. Antihyperalgesic effects of spinal cannabinoids. Eur J Pharmacol 1998; 345 : 145-53.

26. Smith FL, Fujimori K, Lowe J, Welch SP. Characterization of delta9-tetrahydrocannabinol and anandamide antinociception in nonarthritic and arthritic rats. Pharmacol Biochem Behav 1998; 60: 183-91.

27. Ochoa JL, Yarnitsky D. The triple cold syndrome. Cold hyperalgesia, cold hypoaesthesia and cold skin in peripheral nerve disease. Brain 1994; 117: 185-97. 